\title{
Valoración del perfil docente rural desde el proceso formativo y la práctica educativa
}

\section{Assessment of the Rural Teacher's Profile from the Perspective of the Training Process and the Educational Practice}

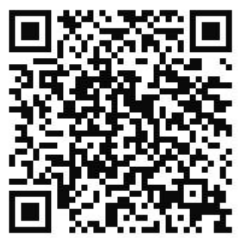

\author{
Andrea Ramírez-González ${ }^{1}$ \\ Universidad Nacional \\ Centro de Investigación y Docencia en Educación \\ Heredia, Costa Rica \\ aramgo3176@gmail.com
}

Recibido 12 de octubre de 2014 • Corregido 27 de junio de 2015 • Aceptado 31 de julio de 2015

\begin{abstract}
Resumen. Este artículo presenta los resultados de una evaluación del perfil del personal docente rural desde la práctica educativa. La bibliografia manifiesta diferentes planteamientos de perfil por funciones, características, rasgos y competencias en los que se consignan aspectos esenciales que lo conforman tales como habilidades, destrezas, saberes, actitudes y valores. En consecuencia, para evaluar los aspectos que configuran la actuación del personal rural, la práctica educativa es el espacio idóneo que permite contrastar distintas propuestas de este perfil profesional. El proceso investigativo consideró una metodología cualitativa con la aplicación de la técnicas de observación participante y entrevista a profundidad a seis docentes de escuelas unidocentes y dirección 1 de escuelas rurales de Costa Rica, con formación académica universitaria en educación rural. Se valoraron las características pedagógico-comunitarias que presenta la práctica educativa y que, a la vez, posibilitan la inserción en espacios laborales alternativos para la zona rural, para recuperar la información necesaria que permitiera valorar el perfil de docentes participantes.
\end{abstract}

Palabras claves. Perfil docente, educación rural, evaluación curricular.

\footnotetext{
${ }^{1}$ Máster en Evaluación Educativa, Universidad de Costa Rica, Licenciada en Enseñanza de la Matemática, Universidad de Costa Rica. Académica de la División de Educación Rural de la Universidad Nacional de Costa Rica. Investigadora de temáticas relacionadas con el perfil de docentes de secundaria y primara, formación de docentes para el ámbito rural. Dirección postal: San José, Cantón de Mora, Costa Rica, 200 metros este y 25 sur Servicentro Delta, Ciudad Colón. Número de teléfono o celular: (506) 22490472, (506) 88112473.
} 
doi: http://dx.doi.org/10.15359/ree.19-3.9

URL: http://www.una.ac.cr/educare

CORREO: educare@una.cr

\begin{abstract}
This paper presents the results of an evaluation of the profile of rural teachers from the perspective of the teaching practice. The literary sources show different profile approaches that are based on the functions, characteristics, traits, and skills and where the essential aspects of the profile such as skills, abilities, knowledge, attitudes, and values are determined. Consequently, the teaching practice is the ideal context to assess the aspects that shape the rural teacher performance since it allows us to compare the various profile approaches. For the research process, a qualitative methodology was applied, through participant observation techniques and an in-depth interview, to six teachers with university studies in rural education of schools classified as single-teacher and administration 1, located in rural areas of Costa Rica. To retrieve the necessary information to assess the profile of the participants, this research examined the pedagogical-community characteristics of the educational practice, which, at the same time, allow the teacher insertion in alternative work spaces in the rural areas.
\end{abstract}

Keywords. Teacher profile, teaching practice, rural education.

Eltema del perfil docente rurales partefundamental de toda propuesta curricularformativa, orientada hacia los procesos de enseñanza y aprendizaje en la zona rural. En este sentido, la bibliografía manifiesta diferentes planteamientos de perfil por funciones, características, rasgos y competencias en los que se consignan aspectos esenciales que lo conforman tales como habilidades, destrezas, saberes, actitudes y valores, de tal manera que configurarán la actuación o desempeño docente rural. De esta manera, el espacio de praxis práctica en el entorno educativo es el idóneo para contrastar y auto-reflexionar sobre las distintas propuestas del perfil a partir del ejercicio de la profesión y, con ello, obtener una mirada de este perfil que permita enriquecer la labor en el espacio educativo y que, a la vez, destellen, a partir de las acciones que realiza en la práctica educativa, posibles nichos laborales que tomen en cuenta la formación universitaria en educación rural.

En ese sentido, esta investigación consideró el perfil profesional propuesto en el plan de estudios de la carrera de Licenciatura en Educación con énfasis en Educación Rural de la Universidad Nacional como referente curricular para valorar la coherencia entre el perfil profesional de esa carrera y la práctica educativa del personal docente rural, a partir de las características pedagógico-comunitarias de seis docentes que laboraban en escuelas públicas rurales de los cantones de Sarapiquí, Heredia, Alajuela y Puriscal.

Las preguntas que orientaron esta investigación fueron las siguientes:

- ¿Cuál es la coherencia entre el perfil profesional de la carrera Licenciatura en Educación con énfasis en Educación Rural de la Universidad Nacional y la práctica educativa del personal docente rural?

- ¿Cómo valoran los docentes y las docentes su formación académica en relación con su propia práctica educativa? 
- ¿Cuáles características pedagógico-comunitarias presenta la práctica educativa del personal docente rural en la actualidad?

- ¿De qué manera el perfil del personal docente rural posibilita su inserción en espacios laborales alternativos?

En el proceso investigativo, se aplicaron las técnicas cualitativas: entrevista a profundidad y observación participante para recuperar la información necesaria que permitiera evaluar el perfil docente de sus participantes.

\section{Referentes teóricos}

\section{Fundamentos históricos para la formación del personal docente rural en Costa Rica}

Las bases históricas constituyentes de la educación rural en Costa Rica y de la formación de docentes vinculados con ella se centran en realidades económicas, políticas, sociales, culturales de nuestro país, que marcaron diversas acciones importantes desarrolladas por los gobiernos con el fin de mejorar la educación rural. A manera se síntesis, sin entrar en el contenido de esas acciones, se mencionan, a continuación, algunas de estas que en distintos periodos de la historia de Costa Rica y con el impulso de educadores como Mauro Fernández, Roberto Brenes Mesén y Omar Dengo Guerrero (quienes con una visión futurista realizaron aportes a la educación costarricense), las cuales sirvieron como bases para cimentar los aspectos que caracterizan las ideologías educativas a lo largo de la historia costarricense (ver figura 1).

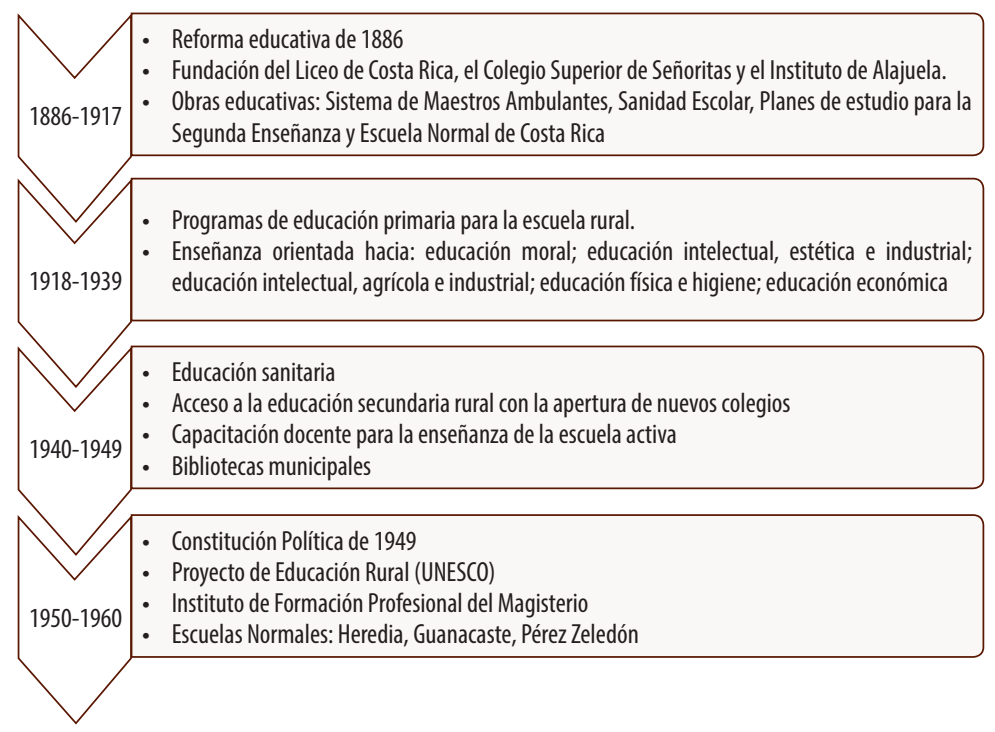

Figura 1. Acciones educativas para mejorar la educación rural: 1886-1960. Referentes teóricos de historia de la educación de Costa Rica 1886-1960. 
doi: http://dx.doi.org/10.15359/ree.19-3.9

URL: http://www.una.ac.cr/educare

CORREO: educare@una.cr

Sumado a las propuestas de los educadores mencionados, es de vital importancia enfatizar la declaratoria realizada en el gobierno de don Jesús Jiménez en 1869, que aprueba la "Ley de Educación", la cual obliga a toda la población costarricense a asistir a la escuela primaria en forma gratuita, pues tiene una influencia profunda en el desarrollo de la vida democrática de nuestro país.

De acuerdo con Dengo (1995), en Costa Rica la formación de docentes en primaria, se inició con la Escuela Normal en 1914 con un plan de seis años que consistía en tres años en estudios generales y otros tres para estudios especializados o pedagógicos. La finalidad de la Escuela era becar estudiantes de zonas rurales de las provincias para que, una vez graduados, se incorporaran al magisterio nacional en sus lugares de procedencia. Asimismo, se convirtió en el centro intelectual y artístico de la ciudad de Heredia y del país por la implementación de nuevas corrientes pedagógicas de la educación de la época.

La creación de la Universidad de Costa Rica en 1940 integró las escuelas de educación superior existentes en la Escuela de Pedagogía, así como la formación profesores de segunda enseñanza en las Facultades de Ciencias, Filosofía y Letras, otorgó el grado de licenciatura para la enseñanza media en las áreas de la ciencia, historia y geografía y filosofía y letras mediante planes de estudios que contemplaban, además de la especialidad, materias pedagógicas.

Con la reforma académica de la Universidad de Costa Rica en 1957, se transformó la Escuela de Pedagogía en la Facultad de Educación para albergar la formación de docentes para la enseñanza primaria y enseñanza media.

En el año 1968 la Escuela Normal se transformó en la Escuela Normal Superior, al incorporar la formación de profesores y profesoras de secundaria. Esta circunstancia cambió en el año 1973 con la creación de la Universidad Nacional (UNA), como respuesta a las necesidades educativas y problemas sociales que enfrentaba la Costa Rica de esa época.

De acuerdo con Aguilar y Angulo (2007), la Escuela de Educación de la UNA tuvo a cargo la formación de docentes mediante dos ofertas curriculares: una denominada "Plan regular" y la otra "Planes de seguimiento". La primera estaba dirigida a las carreras de preescolar, educación especial, primer y segundo ciclos, con un horario diurno entre semana. Mientras la segunda se denominó "Planes de seguimiento" con un modelo que contenía cursos intensivos en las vacaciones escolares y de seguimiento una vez cada mes, en el período de invierno. La población estudiantil de esta última estaba constituida por maestras y maestros en servicio de zonas rurales del país, por lo que la experiencia pedagógica era un insumo necesario en la formación profesional. Los planes de seguimiento estuvieron vigentes de 1973 hasta 1987.

Para Aguilar y Angulo (2007), los planes de seguimiento sentaron las bases en la formación de docentes rurales en la División de Educación Rural de la Universidad Nacional, por la siguiente razón: 
doi: http://dx.doi.org/10.15359/ree.19-3.9

URL: http://www.una.ac.cr/educare

CORREO: educare@una.cr

En el aula universitaria se tomaba en cuenta la práctica pedagógica que estaba realizando el docente y a partir de ella se daba la construcción del conocimiento, se promovía la utilización de recursos del medio, tales como: la naturaleza, los desechos, los recursos humanos. El maestro era un facilitador de los procesos de la organización comunal. (Aguilar y Angulo, 2007, p. 11)

De acuerdo con Solano, Van Kampen y Ovares (2003), los "Planes de seguimiento" fueron una oportunidad para que las personas de zonas rurales lograran finalizar su formación mediante la titulación de docentes de primaria.

En el año 1984, el Ministerio de Educación Pública y la Universidad Nacional se interesaron por la carencia de docentes rurales con título y suscriben un convenio para la formación de quienes estaban en servicio, de tal forma que la preparación académica del estudiante le otorgaba estabilidad laboral en la institución educativa a la que pertenecía. La estrategia descrita originó el Proyecto de Educación Rural (PER), que consideró la experiencia adquirida en la modalidad que desarrolló de los "Planes de seguimiento". La finalidad del PER estaba vinculado con "un plan social y participativo, valorativo del contexto real de la comunidad educativa y de la acción del maestro como líder comunitario" (Aguilar y Angulo, 2007, p. 15).

Entre los años 1986 y 1988 se creó el denominado "Plan de Emergencia de Formación de Docentes", con una oferta de estudios en el nivel de diplomado. Ello solventaba la formación de docentes requeridos en ese instante por el sistema educativo costarricense. Su modalidad era presencial y a distancia.

Posterior al año 1993, la División de Educación Rural ha ofertado un plan de estudios en los niveles de diplomado, bachillerato y licenciatura en la especialidad de Educación Rural dirigido a la formación de docentes para que laboren en el I y Il ciclos de la Educación General Básica.

En la actualidad la carrera de la Universidad Nacional. División de Educación Rural (s. f.), se titula "Licenciatura en Educación con énfasis en Educación Rural, I y II ciclos". El plan de estudios de esta carrera concibe como áreas curriculares la pedagógica, la disciplinar, la aplicativa y la ruralidad contempladas como ejes centrales en los diferentes cursos que componen la malla curricular.

El área pedagógica se refiere al conjunto de conocimientos que tiene su propio objeto de estudio en el hecho educativo; su propio método y algunos fundamentos en las ciencias sociales y en las ciencias naturales.

La disciplinar contempla los conocimientos que las disciplinas aportan a la educación general básica en I y ll ciclos, de tal manera que se incorporan a la práctica mediante experiencias y confrontaciones de teorías, enfoques, conceptos y paradigmas. 
doi: http://dx.doi.org/10.15359/ree.19-3.9

URL: http://www.una.ac.cr/educare

CORREO: educare@una.cr

El área aplicativa posibilita la articulación de los conocimientos adquiridos en las otras disciplinas descritas, con la realidad de la escuela rural, de tal manera que se logren construir alternativas metodológicas y didácticas para estas instituciones.

El perfil profesional se configura a partir de las áreas disciplinares y las competencias que el egresado debe asumir en la docencia rural. En la tabla 1 se presentan, detalladamente, las competencias.

Tabla 1

Competencias del perfil profesional de la Licenciatura en Educación con énfasis en Educación Rural, I y ll ciclos de la Universidad Nacional

\begin{tabular}{|c|c|}
\hline Área disciplinar & Competencias \\
\hline \multirow[t]{2}{*}{ Ruralidad } & $\begin{array}{l}\text { Desarrollar, a partir de su concepción de solidaridad y con sustento en la convicción de que la educación es un vehículo } \\
\text { eficaz para el logro del crecimiento humano, una práctica pedagógica que garantice la comprensión del mundo rural y } \\
\text { la construcción de una pedagogía en este ámbito, mediante la participación de la comunidad en el mejoramiento de la } \\
\text { condición de vida de sus habitantes, en un marco de respeto hacia las personas, sus manifestaciones culturales y su } \\
\text { ambiente. }\end{array}$ \\
\hline & $\begin{array}{l}\text { Promover en la comunidad educativa, acciones que garanticen la adecuación del currículo al área rural, por medio } \\
\text { del desarrollo de procesos de enseñanza y aprendizaje acordes con las necesidades y demandas educativas de las } \\
\text { comunidades rurales de Costa Rica. }\end{array}$ \\
\hline Pedagógica & $\begin{array}{l}\text { Mostrar en su desempeño habilidades, el compromiso con los valores que garanticen un ambiente de relaciones humanas } \\
\text { solidarias en la comunidad y en la escuela; así como la existencia de la armonía y la sostenibilidad con el ambiente natural y social. }\end{array}$ \\
\hline Disciplinar & $\begin{array}{l}\text { Conocer y dominar las teorías educativas y los contenidos didácticos de I y II ciclos, sustentadores de la práctica } \\
\text { pedagógica, que responde a las necesidades de las comunidades rurales. }\end{array}$ \\
\hline \multirow[t]{2}{*}{ Aplicativa } & $\begin{array}{l}\text { Incorporar, a la dinámica de la escuela rural y al aprendizaje de los niños y de las niñas el trabajo investigativo como } \\
\text { reflexión y análisis de la realidad cotidiana y de los contenidos curriculares. }\end{array}$ \\
\hline & $\begin{array}{l}\text { Fungir como elemento que coadyuda a promover cambios en la interpretación de los hechos enfatizados en las } \\
\text { características propias del contexto rural y relacionados con la realidad global. }\end{array}$ \\
\hline
\end{tabular}

Esta carrera, está diseñada para que quien se gradúe pueda desempeñarse como docente en I y II ciclos en Educación General Básica, en cualquier escuela del país, pública o privada. La formación recibida le da ventajas sobre otros educadores y educadoras para trabajar en escuelas ubicadas en las zonas rurales, sean estas unidocente o multigrado.

En el diplomado de la profesión mencionada, se hace énfasis en el desarrollo de características y rasgos concretados en las nociones conceptuales y procedimentales, las cuales permiten una adecuada incorporación del estudiantado al campo laboral docente en una temprana etapa de su formación profesional, así como un acercamiento a la pedagogía rural y a sus diversas aplicaciones en el currículo. 
doi: http://dx.doi.org/10.15359/ree.19-3.9

URL: http://www.una.ac.cr/educare

CORREO: educare@una.cr

Por otra parte, en el bachillerato se enfatizan en los conceptos y procedimientos de facetas relacionadas con la ruralidad en la pedagogía. Por último, en el nivel de licenciatura, se profundiza en las vertientes de lo rural relacionado con lo pedagógico conjuntamente con la investigación. Ello posibilita que el personal profesional desarrolle capacidades, para una mejor observación de los procesos de aula y de las circunstancias sociales de la comunidad, en la elaboración de métodos educativos en la escuela, la familia y la comunidad.

Durante la carrera, el estudiantado en formación debe realizar sus prácticas pedagógicas en las escuelas de la zona rural, principalmente en aquellas designadas como unidocentes y de dirección1. Asimismo, el trabajo final de graduación, en el nivel de licenciatura, se debe hacer en una zona rural en la que la escuela y la comunidad funjan como fuentes de información primaria en la investigación. Conjuntamente con las tareas descritas, se debe participar en giras pedagógicas articuladoras de los cursos del plan de estudios de cada ciclo, con el propósito de acercarse a la escuela. Los proyectos comunitarios de educación no formal en la zona rural son relevantes en la conclusión del plan de estudios.

La formación universitaria también capacita al personal docente desde su trabajo en la escuela, con el fin de que realice actividades académicas en educación, tendientes a desarrollar currículos para el mejoramiento de las condiciones de vida de las poblaciones de las comunidades rurales. Ello, mediante procesos de organización comunal que se llevan a cabo en espacios gubernamentales o no.

\section{Elementos para la conformación del perfil del personal docente rural}

Sin el afán de hacer un análisis histórico de algunos aportes y perspectivas que asumen diversos estudios en relación con el perfil del personal docente rural, es posible determinar en la bibliografía algunas investigaciones y referentes teóricos que hacen valiosos aportes por caracterizar aquellos rasgos, funciones, competencias, atinentes a la formación y al ejercicio de la educación rural, de tal manera que el perfil del personal docente rural, de acuerdo con la propuesta de Thomas y Hernández (2005), se concibe como aquella persona que explora nuevos caminos pedagógicos que potencian, en el estudiantado, la curiosidad, la originalidad; un ambiente de cooperación; valores que posibilitan en sus estudiantes el desarrollo de personas críticas y respetuosas de la diversidad.

En este sentido, la labor pedagógica debe adaptarse a los cambios que las zonas rurales enfrentan, por lo que la planificación de sus actividades requiere de docentes rurales con una posición flexible, proactiva, real y útil para el estudiantado y, por ende, para la comunidad misma.

Autores como Harris, Holdman, Clark y Harris (2005) promueven la idea de que la enseñanza en la escuela rural se basa en un perfil equilibrado de docente en el que se articulan varios aspectos relacionados con una enseñanza desde un enfoque significativo. En este sentido, el contexto 
doi: http://dx.doi.org/10.15359/ree.19-3.9

URL: http://www.una.ac.cr/educare

CORREO: educare@una.cr

cultural se aprecia como parte importante de las estrategias de enseñanza y del desarrollo para las comunidades con estudiantes y familias. Además, son relevantes las oportunidades de aprendizaje y diferencias individuales de estudiantes; el pensamiento crítico y la resolución de problemas mediante el uso de las tecnologías; la comunicación y las relaciones interpersonales.

En este sentido, un perfil docente rural retoma aspectos de la ocupación, del desempeño, sus cualidades y las características de su personalidad en relación con miembros de la comunidad, así como de la formación profesional para la docencia en la ruralidad, por lo que su práctica educativa se configura a partir de características profesionales de las dimensiones académica, personal, ocupacional, fundamentadas en la interacción con la comunidad, los padres y madres de familia y otras instancias gubernamentales o no, influyentes en los procesos educativos.

En consecuencia, el ejercicio de la docencia en las zonas rurales requiere de una formación universitaria en permanente acercamiento con la escuela rural y una práctica profesional que permita el conocimiento de las dinámicas que caracterizan la labor docente. Por ello, Miller (1991) cree necesario tener prácticas en la escuela rural seguidas del desarrollo de capacidades en el plano curricular enlazadas con la planificación, la organización de la clase, la enseñanza individualizada, la recolección de recursos y materiales.

Desde otra óptica, la propuesta de Boylan (2003), referida a la preparación de docentes para la escuela rural, sugiere que los programas de formación deben incluir cursos y actividades académicas en relación con la pedagogía para múltiples grupos de edad; la organización y planificación de la clase para el aula rural; estrategias de gestión y eficacia del tiempo; responsabilidades administrativas; acceso de recursos; estrategias de participación e interacción con la comunidad; influencia de la comunidad en los procesos de enseñanza y aprendizaje en el medio ambiente; desarrollo de apoyo con redes de comunicación con docentes rurales para conocer sus experiencias y comunidades.

Por su parte, Thomas y Hernández (2005) mencionan que la formación de docentes rurales requiere de cursos que promuevan cualidades que fortalezcan el desempeño en la escuela rural, unidas con la curiosidad y la originalidad. Se fomenta un ambiente de cooperación y solidaridad en el aula; además la visión crítica y la autocrítica; la confianza en sí; la responsabilidad y la independencia.

Algunos referentes sobre el perfil del personal docente rural también consideran aspectos relacionados con la labor en la escuela multigrado o unidocente, es decir, conformadas por un solo grupo de estudiantes de diferentes edades y niveles, por lo que exige un desempeño acorde con las particularidades descritas.

En este sentido, Bustos (2007) señala que la actuación docente en la escuela multigrado requiere de una distribución de tiempo de las tareas, para ello recomienda una planificación meditada, sistematizada del quehacer escolar, pues la improvisación conlleva a que el volumen de trabajo sea mayor, provocando insatisfacción y ansiedad del propio desempeño. 
doi: http://dx.doi.org/10.15359/ree.19-3.9

URL: http://www.una.ac.cr/educare

CORREO: educare@una.cr

Asimismo, se le confiere importancia a las "agrupaciones flexibles" en la organización del estudiantado, especialmente en el aula multigrado o unidocente, en términos de que los agrupamientos no se conformen necesariamente por el nivel oedad cronológica correspondiente a cada estudiante, sino por las capacidades de cada quien. Esto invita a una flexibilización del currículo, de la enseñanza, pues implica también cambios en las tareas y en las actividades de aprendizaje en relación con los programas de estudio, según el nivel educativo.

Para Wragg (1984, citado por Miller, 1991), la conformación del perfil docenterural se relaciona con las habilidades necesarias para quienes laboran en la escuela multigrado o unidocente:

- Organización del aula, según los recursos utilizados para la enseñanza en un ambiente físico que facilite el estudio independiente.

- Desarrollo de programaciones en el aula, con rutinas promotoras de la responsabilidad del alumno y la alumna de su propio estudio.

- Fomento del trabajo cooperativo y autoaprendizaje de acuerdo con las necesidades estudiantiles, a partir de la planificación, el desarrollo y la realización estrategias de enseñanza.

- Entrega de la docencia con métodos y estrategias para organizar el grupo que aprende en los niveles del grado; especialmente aquellos que desarrollan la interdependencia y la cooperación entre estudiantes.

- Estudio autodirigido para el desarrollo de habilidades y estrategias en los alumnos y alumnas, quienes tienen un nivel alto en independencia y eficacia en el estudio individual o en la combinación con otros y otras estudiantes.

- Formación de pares quienes potencien el desarrollo de habilidades y de rutinas, de tal manera que estudiantes sirvan como "docentes" que permiten mediar el proceso de enseñanza con lo que se propicia la generación de aprendizajes de estudiantes en los distintos niveles del grado.

Otra perspectiva la aportan Solano et al. (2003), quienes sintetizan las tareas del docente rural en diversas actividades relacionadas con la planta física de la escuela; el trabajo de comités, apoyo administrativo y financiero; los padres y madres de familia; la enseñanza y aprendizaje de sus estudiantes. Estas actividades se enmarcan dentro de un quehacer educativo orientado a la gestión administrativa, proyección de la escuela hacia la comunidad, el proceso de enseñanza y aprendizaje y la relación familia-escuela-comunidad.

De esta manera, se rescata que la formación del educador y educadora rural no se basa en competencias circunscritas para la enseñanza en aula escolar, sino que también se requiere de un perfil que refleja otros aspectos como la gestión administrativa y curricular, el 
doi: http://dx.doi.org/10.15359/ree.19-3.9

URL: http://www.una.ac.cr/educare

CORREO: educare@una.cr

clima de aula, la participación e interacción con la comunidad, la comunicación, cualidades y rasgos de la personalidad.

\section{Evaluación curricular}

La evaluación curricular se concibe como corte y articulación entre los discursos del currículo y de la evaluación, en el contexto de las ciencias humanas. Por eso, se entiende el currículo de la siguiente manera:

Síntesis de elementos culturales (conocimientos, valores, costumbres, creencias hábitos) que conforman una propuesta político educativa pensada e impulsada por diversos grupos y sectores sociales; propuesta conformada por aspectos estructurales-formales y procesales prácticos, así como dimensiones generales y particulares que interactúan en el devenir de los currícula en las instituciones sociales educativas. (De Alba, 1991, p. 62)

Desde esta perspectiva, la evaluación curricular parte de la noción de totalidad epistemológica referida a una posición constante frente a la realidad social cambiante y dinámica, que posibilita delimitar un campo de problematización y observación, mediante una teoría del problema por evaluar, lo que presupone una perspectiva teórico-conceptual a partir del cual se construye el objeto y del cual se infiere la definición metodológica y técnica asumida. Todo ello vinculado a ciertas características teórico-axiológicas como elementos constitutivos de la evaluación para poder conformar el juicio que se realiza al objeto de evaluación.

De Alba (1991) define el carácter teórico de la evaluación, como una "comprensión conceptual desde una posición teórica que permite la comprensión de lo evaluado" (p. 95). Para esta autora, la importancia del sustento teórico en la evaluación radica en que se disminuye la posibilidad de recurrir a procesos de enjuiciamiento y no de evaluación.

El carácter axiológico de la evaluación (De Alba, 1991, p. 98) se refiere al "conjunto de valores a partir de los cuales se analiza el objeto-sujeto por evaluar".

Desde la perspectiva de totalidad asumida por De Alba (1991), se confiere especial interés a la hermenéutica-dialéctica como opción metodológica del enfoque cualitativo que "centra la atención en la comprensión (verstehen) más que en la explicación causal (erklären)” (p. 151).

Desde esta visión teórica de evaluación curricular, esta investigación se propuso evaluar el perfil docente como uno de los elementos fundamentales del currículo, partiendo de los referentes teóricos en relación con el perfil, la práctica educativa del personal docente en contexto de ruralidad y de la formación profesional en el ámbito de la educación rural. 
doi: http://dx.doi.org/10.15359/ree.19-3.9

URL: http://www.una.ac.cr/educare

CORREO: educare@una.cr

\section{Método}

En esta investigación del campo especializado de la educación rural, se analizó la práctica educativa como referente del perfil del personal docente en este medio, que se enriquece al transformar la concepción teórica asumida a la interpretación del perfil, en la realidad de la práctica educativa.

Para comprender el perfil docente rural, fue necesario dilucidar valores, sentimientos, intereses, desempeño, actuación, concepción educativa, mediante la comprensión, la construcción e interpretación de significados derivados de la actuación del personal docente en las realidades rurales a partir de las interacciones constantes en los contextos educativos y prácticas educativas cotidianas. Además, con el propósito de analizarlos, se tomaron, de la práctica educativa del personal docente rural, elementos de las competencias que configuran el perfil de formación de la carrera de Educación Rural, en relación con el contexto natural del ejercicio de la profesión.

La escogencia de los docentes y las docentes participantes para la recuperación y construcción de la información se realizó con base en su formación en educación rural, que laboraran en instituciones de I y II ciclos de acuerdo con las características institucionales, geográficas y organizativas. Se seleccionaron seis instituciones educativas públicas de I y II ciclo ubicadas en zonas rurales pertenecientes a las direcciones regionales educativas del Ministerio de Educación Pública en Puriscal, Sarapiquí, Heredia y Alajuela. En total, participaron seis docentes, tres mujeres y tres hombres, quienes laboraban en alguna de las instituciones de estas regiones.

Para analizar la información, se requirió de suficiente pericia para decidir en qué determinado momento de la evaluación se logró el nivel de interpretación óptimo y la rigurosidad metodológica vinculados con la producción de la información.

Se organizaron las entrevistas a profundidad, las observaciones de práctica educativa y registros del diario de campo como documentos electrónicos de texto para: a) la revisión de los datos que permitió categorizar la información recuperada; b) la obtención de resultados mediante las interpretaciones, explicaciones y conjeturas, c) la verificación de conclusiones.

A partir de la guía de tópicos que guiaron las técnicas cualitativas aplicadas en este estudio, a saber, la entrevista y la observación, por conveniencia analítica se consideraron cuatro categorías:

- Significaciones de la carrera en la formación profesional y personal según las valoraciones que hacen los docentes y las docentes a la carrera de educación rural, así como de la adaptación que se requiere para laborar en ese entorno.

- Elementos que intervienen en la práctica educativa del personal docente rural en el aula escolar. 
doi: http://dx.doi.org/10.15359/ree.19-3.9

URL: http://www.una.ac.cr/educare

CORREO: educare@una.cr

- La práctica educativa y sus relaciones en el contexto institucional y social que contempla aspectos sobre la relación familia-escuela-comunidad, gestión para la organización comunitaria y la situación social de la comunidad rural.

- Las oportunidades del personal docente rural en la actualidad, referidas a los espacios laborales tradicionales y alternativos.

La información producida se contrastó y se comparó con la teoría y la formación profesional en educación rural en la Universidad Nacional, recibida por el grupo de participantes para obtener hallazgos consistentes y válidos en la investigación, de tal manera que se lograron interpretaciones válidas a partir de las coincidencias o relaciones entre los datos producidos de las entrevistas a profundidad y las observaciones sistematizadas.

\section{Resultados}

\section{Significaciones de la carrera en la formación profesional y personal}

La educación costarricense, por las características geográficas y sociales del país, cuenta con escuelas en las zonas rurales del país que requieren docentes con formación universitaria para laborar en el área de primaria. Desde este punto de vista, la División de Educación Rural ha estructurado un programa de formación de docentes en algunos lugares alejados de la Sede Central "Campus Omar Dengo" en Heredia.

De acuerdo con los registros de cantidad de personas graduadas de la carrera de Licenciatura en Educación con énfasis en Educación Rural de la División de Educación Rural de la UNA, desde el año 1990 hasta el 2006 de esta carrera se han graduado docentes en los niveles de Diplomado, Bachillerato y Licenciatura en diferentes regiones del país, entre ellas: Liberia, Cañas, San Carlos, Puntarenas, Los Santos, Limón, Siquirres, Buenos Aires de Puntarenas, Bribri y Puriscal. Además, en la Sede Central en Heredia, se han atendido y graduado dos mil quinientos estudiantes aproximadamente, provenientes de zonas rurales.

Engeneral, la formación universitaria recibida en la División de Educación Rural es altamente valorada desde el punto de vista académico y desde la relación del aprendizaje con el medio natural que rodea a la escuela vinculada con esta orientación. Consecuentemente, durante la carrera se confirió la oportunidad de aprender perspectivas teóricas, para la enseñanza primaria en su contexto natural.

En la carrera se realizaron experiencias prácticas fortalecedoras de la relación teoría-práctica aplicada en la escuela rural. La finalidad es que la persona docente mostrara sus habilidades y fortalezas como futuro o futura profesional. Cabe destacar que algunas de esas experiencias prácticas han sido incorporadas como parte de la práctica educativa en el aula escolar rural. 
doi: http://dx.doi.org/10.15359/ree.19-3.9

URL: http://www.una.ac.cr/educare

CORREO: educare@una.cr

Otra docente valoró que el grupo de docentes de la carrera de Educación Rural ha laborado en educación primaria o en las actividades de investigación y extensión en la Universidad Nacional, por lo que durante el desarrollo de los cursos mostraron conocimientos acerca de la realidad educativa de la escuela rural. Por lo tanto, lograron vincular, desde la mediación pedagógica en el aula universitaria, sus experiencias y aprendizajes para propiciar la relación teoría-práctica.

Es importante señalar que la interacción entre estudiantes en formación y docentes de de la universidad, en las diferentes actividades académicas, marca definitivamente el actuar profesional futuro. El impacto positivo o negativo de esta circunstancia durante las lecciones de los cursos, en la forma de conducirse y de dirigirse del personal docente que media el proceso hacia el estudiantado, se considera un modelo por seguir y repetir posiblemente en la escuela rural. De ahí, que la docencia universitaria merece especial atención por la influencia producida en la formación del futuro profesional.

Para mejorar la preparación académica docente universitaria en educación rural, uno de los participantes sugirió la actualización permanente en temas atinentes a la educación primaria para este contexto, con bibliografías novedosas, conocimientos básicos de andragogía, sin dejar de lado el contacto con la realidad de la escuela.

Un aspecto que exalta la pertinencia formativa en educación rural está referido al desempeño en la escuela unidocente de la zona rural, pues en esta se atienden estudiantes de distintos niveles de manera simultánea, es decir, los niños y las niñas de primero a sexto grado comparten un mismo espacio físico y formativo para recibir sus lecciones de todas las materias que conforman el currículo nacional. En consecuencia, la labor unidocente requiere de un conocimiento para el manejo de grupo, el planeamiento didáctico y la gestión administrativa de manera particular. No obstante, se indica que durante la carrera es necesario fortalecer cursos en materia de administración educativa, ya que en la escuela rural asumen la responsabilidad de la dirección del centro educativo.

La oportunidad de acercamiento a la escuela rural durante el proceso de formación profesional y luego como parte de la labor docente es una actividad que permite visualizar la realidad educativa a la que tiene que adaptarse, en un contexto específico.

De acuerdo con los registros de esta investigación, las comunidades rurales de las escuelas participantes se caracterizan por:

- Trabajo de campo en fincas destinadas principalmente a la agricultura y ganadería.

- Familias de bajo nivel educativo formal y pocos recursos económicos.

- Condiciones socioeconómicas del estudiantado que limitan la inversión para estudiar, como la compra de materiales, la alimentación y el transporte.

- Cada comunidad rural tiene formas de convivencia, problemáticas, costumbres, conflictos entre lasfamilias, clima yalimentación, que la hacen particulary diferentea otras comunidades. 
doi: http://dx.doi.org/10.15359/ree.19-3.9

URL: http://www.una.ac.cr/educare

CORREO: educare@una.cr

Por ello, es necesario que el docente o la docente conozca e indague con personas de la comunidad acerca de sus costumbres, principales actividades económicas, recreativas, fuentes de trabajo, para que tenga un panorama general de los aspectos sociales, económicos y culturales de la zona en que labora y, de esta manera, proyectar el tipo de ciudadana y ciudadano que habita en ese lugar y de qué manera contribuirá su labor en la formación de esas personas.

Cada docente, para adaptarse al medio rural, requiere conocer del clima, la topografía del lugar, infraestructura de la escuela, para que reflexione sobre la adecuación o los ajustes tanto personales como académicos durante su estadía en la comunidad, ello incluye vestir con decoro, utilizar indumentaria acorde con las condiciones climáticas de la comunidad y las costumbres locales, con el fin de lograr la aceptación como miembro más de la comunidad rural.

Ese proceso de adaptación de la persona docente en el medio rural puede transcurrir con facilidad, para quienes procedan de zona rural o constituirse en una situación complicada, por cuanto algunas zonas no cuentan con servicios básicos de electricidad, agua potable, transporte o red vial adecuada. En este sentido, la adaptación al entorno es reconocida como una competencia genérica interpersonal necesaria para que las personas logren una buena interacción con las demás, lo que significa que "la destreza para adaptarse a las nuevas circunstancias, manteniendo el estado de equilibrio en el nuevo contexto, cuando las condiciones son adversas" (Villa y Poblete, 2007, p. 219).

\section{Elementos que intervienen en la práctica educativa del personal docente rural en el aula escolar}

\section{Planificación de la enseñanza}

En la actualidad, el currículo básico nacional se centra en áreas o asignaturas como español, matemáticas, ciencias, estudios sociales, lengua extranjera y otras materias complementarias como educación religiosa, educación física, agricultura, artes plásticas, lo que evidencia un programa de estudio para cada asignatura, un aprendizaje y un conocimiento fragmentado.

En una de las observaciones de lección fue posible identificar espacios de aprendizaje llamados "rincones", para cada una de las asignaturas. En estos, hay algunos carteles alusivos a la materia, por ejemplo: en español, el alfabeto; en estudios sociales, dibujos realizados por los niños y las niñas sobre el encuentro de culturas.

No obstante, en los años 90, el MEP propuso una planificación de la enseñanza para el I ciclo de la Educación General Básica, en la que se posibilita una integración del currículo a través de unidades de aprendizaje que correlacionan temáticas de las disciplinas básicas; una de las colaboradoras opinó que aunque ya no es directriz del Ministerio de Educación Pública (MEP) el planeamiento integrado, esta alternativa sigue vigente para la escuela rural, porque posibilita 
doi: http://dx.doi.org/10.15359/ree.19-3.9

URL: http://www.una.ac.cr/educare

CORREO: educare@una.cr

que desde un eje orientador o situación-problema no solo se pueden articular distintos temas de las asignaturas, sino que se promuevan las relaciones afectivas entre el grupo de pares y sus familias, por lo que es necesario rescatar esta forma de programar la enseñanza y el aprendizaje.

La tendencia de planificación en forma integrada fue sustituida nuevamente por la segmentada en materias básicas, con la adición de algunos elementos curriculares como los ejes transversales, los cuales complementan la preparación de la comunidad estudiantil para la vida.

Por lo tanto, en los planeamientos revisados en esta investigación no se pudo constatar la existencia de actividades que propician un aprendizaje integrado, ni tampoco el uso de libros de texto en los que la propuesta didáctica esté orientada a la integración del conocimiento.

El uso de la computadora es una herramienta tecnológica que los docentes utilizan para estructurar su labor en el aula, por lo que fue posible observar computadoras portátiles o fijas con carpetas de documentos electrónicos, como referencia para los planeamientos didácticos. También, en una ocasión, se logró observar presentaciones en power point como parte de las actividades de mediación.

La planificación didáctica toma como punto de partida las directrices curriculares emanadas por el MEP, orientadas hacia un currículo nacional básico, constituido por asignaturas independientes para cada nivel educativo, las cuales poseen elementos curriculares como objetivos generales y específicos, contenidos, estrategias de mediación, actitudes y valores, aprendizajes por evaluar y la crónica semanal.

Por tanto, los contenidos se organizan multidisciplinariamente, por lo que desaparecen las posibles relaciones entre las disciplinas. Únicamente uno de los participantes se desvincula de la directriz ministerial, al incorporar otras asignaturas como parte del currículo desarrollado en su escuela, por su propia convicción profesional y educativa. Por ello, asume una modificación en las materias que desarrolló, como alternativa de flexibilidad curricular necesaria en la escuela rural, lo cual demuestra una decisión relevante para atender las necesidades emergentes en la localidad que en evidencia no responden a un interés del MEP.

Asimismo, casi no se visualizan elementos del entorno natural y cultural incorporado en las actividades de mediación, aunque se menciona la gira pedagógica como estrategia didáctica pertinente para la contextualización curricular. Sin embargo, los participantes y las participantes manifestaron interés por recuperar una planificación desde una perspectiva integral en la que se parte de los intereses y de las necesidades estudiantiles.

\section{Recursos didácticos en la mediación pedagógica}

Para propiciar el aprendizaje del estudiantado, cada docente se apropia de diferentes recursos como estrategias, técnicas y materiales didácticos en las actividades de mediación 
doi: http://dx.doi.org/10.15359/ree.19-3.9

URL: http://www.una.ac.cr/educare

CORREO: educare@una.cr

pedagógica para apoyar y complementar el proceso educativo, de tal manera que sean seleccionadas de acuerdo con los objetivos y los contenidos por desarrollar. También es importante considerar las necesidades e intereses de los niños y de las niñas, las oportunidades del ambiente natural y comunitario en que se encuentra inmersa la escuela rural.

Dos estrategias observadas, en todas las visitas a los docentes y las docentes participantes de este estudio, fueron las preguntas y la lectura de textos.

La primera estrategia se utilizó para despertar el interés en el grupo de estudiantes, en diferentes momentos del desarrollo de la lección, por ejemplo, como incentivación para recordar un contenido, en las explicaciones hechas por el docente o la docente sobre la temática estudiada, en las conversaciones docente-estudiante sobre situaciones personales, entre otros. En una de las lecciones observadas se hicieron preguntas tales como: ¿qué es?, ¿cómo se usa?, ¿para qué sirve?, ¿qué tamaño tiene?, ¿qué parecido tiene con...?, ¿por qué sucede?, ¿para quién es?

La lectura de textos es empleada por el grupo docente para sintetizar los contenidos y mejorar el vocabulario de los niños y de las niñas, independiente de la asignatura estudiada. Para ello, se subrayan palabras claves, oraciones importantes, párrafos cortos con ideas principales que se copian en los cuadernos, se hacen resúmenes.

Por otra parte, se constató en esta investigación que se realizan extensas prácticas y cuestionarios para cada uno de los contenidos estudiados como estrategia para repasar lo aprendido. Por ejemplo, en una lección de matemática privan los procedimientos para operaciones fundamentales y problemas de geometría, por lo que hay poca construcción de conceptos matemáticos con uso de material concreto.

Un complemento importante en el proceso de enseñanza y aprendizaje son los materiales didácticos mediante el uso de tecnologías que, en la actualidad, pueden o no diferenciar una escuela urbana de una rural, por cuanto los servicios de telefonía y de electricidad no llegan a todas los centros educativos rurales del país. Por tanto, obtener una computadora con INTERNET, un video bean, un televisor con sistema de televisión por cable, un VHS o DVD, un laboratorio de informática, permite una práctica pedagógica con información actualizada, creativa y vistosa para el estudiantado.

La posibilidad de utilizar un recurso como la computadora permite escuchar y ver cuentos infantiles, juegos de acuerdo con los contenidos de los programas, elaboración de proyectos educativos, entre otros. No obstante, este instrumento en la escuela rural hace que el docente tenga la necesidad de capacitación en el uso de la tecnología y medios audiovisuales. Por esta razón, ella se debe iniciar en los años de preparación inicial universitaria, de manera continua y durante el ejercicio de la profesión. 
doi: http://dx.doi.org/10.15359/ree.19-3.9

URL: http://www.una.ac.cr/educare

CORREO: educare@una.cr

\section{Evaluación de los aprendizajes}

Para conocer los resultados de los objetivos planteados en un nivel escolar, es necesario valorar las actividades de mediación y los conocimientos construidos por cada estudiante con la aplicación de estrategias e instrumentos, de tal manera que la información derivada de estos le permita al docente mejorar su propuesta educativa para el crecimiento personal y académico de la comunidad de aprendizaje. Para ello, cada docente se apoya en el "Reglamento de Evaluación de los Aprendizajes", normativa fundamentada en los preceptos ideológicos del Ministerio de Educación Pública que regula los procesos de evaluación del sistema educativo costarricense, lo cual indica que para este grupo de docentes no se trasciende en el uso de otras estrategias o instrumentos que no tenga un respaldo normativa.

En la revisión de instrumentos evaluativos realizada, se encontraron rúbricas, escalas de cotejo, pruebas escritas, registros de desempeño, orientaciones para la prueba oral y de ejecución, entre otros, empleados por las educadoras y educadores entrevistados. Cabe señalar que no se contempló un análisis de calidad de los instrumentos propuestos por el grupo de participantes.

También se observaron, durante las lecciones, actitudes valorativas de parte del personal docente hacia el estudiantado, desde el punto de vista formativo, por lo que no se califica con un puntaje cada uno de estos aspectos. Por consiguiente, el objetivo es que el grupo de estudiantes mejore en su proceso de aprendizaje mediante la revisión de cuadernos para corregir o reforzar aspectos relacionados con ortografía, redacción, limpieza y orden durante el trabajo cotidiano. Durante la estancia de parte de la investigadora en diferentes lecciones y escuelas, no se observó ninguna estrategia o instrumento de carácter evaluativo en la que se diera una evaluación entre pares; ni tampoco alguna estrategia e instrumento que le permitiera al docente realimentar su función mediadora del proceso desde la valoración del grupo de estudiantes. Esto indicó que no se asume una evaluación en la que participan estudiantes y docentes que permita obtener posibles acciones de mejora en el proceso educativo.

Aunque no se cuestiona la necesidad de los distintos instrumentos, para evaluar el proceso de enseñanza y aprendizaje es una realidad que invierten gran cantidad de tiempo extra, que debe realizar el docente y la docente como parte de la planificación escolar. En ocasiones no es posible cumplir con estas pretensiones en su totalidad, por ello requieren intercambiar propuestas de evaluación con colegas del circuito educativo, de tal forma que sean documentos de referencia para construir los propios, como en el caso de las pruebas. Por lo tanto, es necesario que el personal docente de estas escuelas innove en estrategias e instrumentos que le permitan construir una propuesta evaluativa que sea más amplia, útil, genérica y expedita para una valoración de los aprendizajes más justa desde una posición ética del educador y educadora rural hacia el estudiantado y hacia sí mismo como profesional. 
doi: http://dx.doi.org/10.15359/ree.19-3.9

URL: http://www.una.ac.cr/educare

CORREO: educare@una.cr

\section{Gestión administrativa}

En las escuelas unidocentes y dirección 1, la gestión administrativa es considerada por el MEP como un recargo de funciones, para el que se otorga una baja remuneración económica que no compensa la responsabilidad que demanda, de tal forma que el personal docente combina la labor pedagógica con las obligaciones exigidas por la administración del centro educativo.

Como parte de las funciones del director o directora, se vinculan con el cumplimiento de las solicitudes emanadas de las jerarquías del MEP y de las familias de los estudiantes. En ocasiones ello causa molestias en las familias de estudiantes, porque no comprenden las diversas tareas y compromisos que debe atender como parte de la gestión administrativa.

Las actividades que desarrolla un docente en un puesto directivo son caracterizadas por los objetivos de la institución educativa, algunas de estas se enlazan con la participación en la Junta de Educación y el Patronato Escolar, comités constituidos por familiares de los estudiantes o personas de la comunidad.

Una cualidad del gestor educativo es el liderazgo proyectado dentro o fuera de la escuela rural, que le permite influir sobre las demás personas a partir del trabajo en equipo, de la participación de los demás docentes o miembros de la comunidad, en acciones benéficas para la institución.

\section{La práctica educativa y sus relaciones con el contexto institucional y comunal}

\section{Gestión para la participación comunitaria}

Para el grupo de participantes, algunas de las directrices emanadas por el MEP acerca de la gestión administrativa se llevan a cabo por la escuela, docentes y familias. Sin embargo, en algunas ocasiones se presentan fallas, principalmente respecto a comunicación entre ambas partes, por lo que se muestra algún malestar o problema.

La escuela en la comunidad rural requiere, indudablemente, de la figura docente como profesional, quien fortalece la participación de la comunidad, sus habitantes, los grupos organizados y las empresas que, conjuntamente contribuyen a solventar diversas necesidades originadas en la institución educativa. Esa participación implica acción, responsabilidad, compromiso compartido, para que los proyectos que surjan se conciban como propios para los involucrados. En este sentido, la gestión para la participación de la comunidad involucra distintos actores tales como: el personal docente y administrativo (si lo hubiera), el grupo de estudiantes y su familia, la comunidad y las instituciones gubernamentales o no gubernamentales, y grupos sociales organizados pertenecientes a la comunidad.

De esta manera, cada docente, en el medio rural, lidera procesos de participación comunitaria, desde la formulación y la ejecución de proyectos, según las necesidades detectadas y sobre la base del contexto socio-cultural en el que está inmerso. 
doi: http://dx.doi.org/10.15359/ree.19-3.9

URL: http://www.una.ac.cr/educare

CORREO: educare@una.cr

De esta forma, se toma en cuenta la Ley Fundamental de Educación (Asamblea Legislativa de a República de Cossta Rica, 1957) que en el capítulo IX De la extensión cultural, le atribuye al Ministerio de Educación Pública -en este caso al personal docente como figura que representa a este Ministerio- responsabilidades que promuevan en las comunidades, entre varios aspectos, el nivel cultural, y la protección de los recursos patrimoniales, históricos y artísticos.

Por otra parte, una de las acciones pedagógicas en su gestión administrativa, con respecto a la escuela, se relaciona con la documentación legal en la inscripción del terreno de la escuela en el registro de la propiedad, plano, escritura y la cédula jurídica de ella. Esos son requisitos indispensables, para solicitar cualquier ayuda económica, más aún cuando la comunidad no cuenta con organización grupal como la Asociación de Desarrollo Comunal, que le permita canalizar fondos públicos o donaciones hacia la institución educativa.

Para lograr el acercamiento de la población a la escuela y viceversa, es necesaria la realización de un "diagnóstico comunitario" por parte del personal docente de la escuela, para conocer con más detalle las necesidades, las oportunidades, los servicios con los que cuenta la sociedad rural. Esto les permite adentrarse en la colectividad rural, que es la base para la formulación de un proyecto educativo, que, en palabras de Antúnez (2001), es un instrumento de la gestión vinculado al contexto, estructurado por objetivos y conformación organizativa, con los que se identifica el centro educativo.

La construcción de un proyecto educativo institucional requiere de docentes de la escuela, para conocer y detectar las necesidades de sus estudiantes y de la comunidad en general, con la finalidad de que, desde el planteamiento, se promuevan distintas estrategias, para solventar los requerimientos.

Para cumplir con lo anterior, el grupo de personas entrevistadas argumenta que es necesaria la existencia de la continuidad laboral en la función docente-director en la escuela, al menos durante un año.

El proyecto educativo institucional debe contemplar, como primer elemento esencial, un diagnóstico elaborado por docentes de la escuela, que les permita, junto con las familias y los miembros de la comunidad, conocer y participar de las necesidades e intereses por concretar y desarrollarse en proyectos comunitarios y escolares. En este sentido, el grupo de docentes participantes afirman que la escuela no siempre cuenta con un plan institucional o, por lo menos, no se da a conocer a los miembros de la comunidad educativa, en el caso de que existiera.

Una necesidad manifestada en todas las escuelas participantes y sus comunidades, que podría constituir parte de un proyecto educativo institucional, es la apertura de un centro de cómputo y la apertura de una biblioteca escolar como espacio adecuado para audiovisuales, talleres, computación que complemente el aprendizaje de los niños y las niñas. 
doi: http://dx.doi.org/10.15359/ree.19-3.9

URL: http://www.una.ac.cr/educare

CORREO: educare@una.cr

También, llama la atención la importancia de la promoción de actividades recreativas por parte de la institución educativa, como una de las pocas oportunidades para que la comunidad comparta, se divierta y recupere fondos económicos, con el fin de invertirlos en la ella misma; por ello, la participación docente y de la población en comités es fundamental para el bienestar comunitario-familiar y de la escuela misma, ya que la colaboración se hace sin recibir paga alguna, por lo que todos los dineros que se recaudan son destinados con base en el propósito planteado.

De esta manera, la gestión para lograr una participación comunitaria se basa en un liderazgo compartido con personas u organizaciones que logran proponer proyectos educativos -o de otra índole- que activen a la comunidad en general.

Asimismo, se le confiere a la escuela el poder de convocar y participar en las distintas acciones que se realicen para mejorar las condiciones de vida de las personas de la zona rural, por lo que una actitud proactiva y de responsabilidad debe caracterizar al personal docente que labora en este contexto.

\section{Las oportunidades laborales del personal docente rural en la actualidad}

El personal docente rural en la actualidad requiere ampliar sus opciones laborales, por lo tanto, es necesario que conozca a los empleadores tradicionales y los alternativos; las posibilidades de inserción en otros espacios de trabajo, actividades extraescolares y de educación no formal, en las que puede participar, así como las expectativas de empleo.

El Ministerio de Educación Pública tradicionalmente ha sido la fuente principal empleadora para el docente rural y la mayor alternativa laboral, con la posibilidad de ocupar distintos puestos docentes y administrativos dentro de él, con la titulación universitaria en el área educativa.

En pocas ocasiones se presenta la opción de obtener ocupaciones en otras áreas docentes, como en el caso de la atención de grupos de alfabetización con horarios vespertinos que permiten a personas de la comunidad iniciar o continuar un proceso de alfabetización para aprender a leer, escribir y obtener conocimientos básicos en matemáticas, que les proporcione a estas personas vincularse con su entorno y el mundo con otras herramientas.

Sin embargo, aunque exista disposición del personal docente para conformar estos grupos, no solo por la remuneración económica que puede tener, sino por el interés de mejorar los niveles educativos de las personas integrantes de la comunidad en la que labora, el MEP determina una serie de requisitos administrativos que deben cumplirse para la apertura de una opción educativa de esta naturaleza, privativa de los intereses de la comunidad, lo cual limita las posibilidades reales de llevar a cabo estas iniciativas. En ese sentido, quizás se requiere una organización comunitaria que permita generar procesos autogestionarios y no dependientes del MEP que faciliten el logro de un propósito comunal como lo es la alfabetización de sus habitantes. 
doi: http://dx.doi.org/10.15359/ree.19-3.9

URL: http://www.una.ac.cr/educare

CORREO: educare@una.cr

Para el grupo de entrevistados, son pocas las expectativas laborales, diferentes a las del MEP, a las que pueden optar en la actualidad con el mismo título profesional docente. Algunas expectativas se citan textualmente a continuación:

- Guía turístico rural, para empresas agrícolas que ofrecen tours para mostrar los procesos de producción y en reservas forestales.

- Manejo de grupos.

- Impartir charlas.

- El uso de la pedagogía llevado a profesionales que no son del área educativa.

En cada una de las nuevas alternativas de trabajo presentadas, no explican con precisión cuáles son las funciones, pero sí el porqué, en calidad de docente, se puede incursionar en estas. Se parte del supuesto de que con la formación en docencia rural se desarrollan habilidades y actitudes como liderazgo, comunicación, organización de grupos, planificación, actualización profesional, conciencia ambiental que les permite perfilar el desempeño docente y ver más allá del aula escolar rural como espacio laboral. Estas posibilidades laborales inciden en que se amplíe el objeto de estudio de esta carrera potenciando la educación no formal para que el estudiante en formación viva diversas experiencias que, en un futuro próximo, se conviertan en oportunidades para su desempeño profesional.

\section{Resultados y discusión}

Las valoraciones de la carrera de Licenciatura en Educación con énfasis en Educación Rural, I y II ciclos, hechas por el personal docente participante, son reconocidas como pertinentes y necesarias para el ejercicio de la docencia rural, pues durante los cursos se adquieren los conocimientos esenciales para el desempeño de la educación primaria en estos contextos, particularmente lo relacionado con en el quehacer pedagógico y administrativo de la escuela unidocente o multigrado, lo cual muestra habilidades técnicas del saber procedimental de la competencia de ruralidad y disciplinar del perfil profesional del personal docente rural.

Para el grupo entrevistado, es trascendental que los cursos de la carrera de Educación Rural, en su mayoría, sean teóricos-prácticos, porque posibilitan que el docente se involucre en la dinámica cotidiana de la escuela rural. Los conocimientos teóricos son estudiados en la universidad y llevados a la práctica educativa, para identificar situaciones socioeconómicas, costumbres, tradiciones, problemáticas presentes en las comunidades rurales.

Los espacios de práctica realizados durante la carrera permiten el acercamiento y la sensibilización de la persona a la realidad de las escuelas, por lo que es conciente de la importancia de adaptarse al medio rural en el ejercicio de la profesión, con la finalidad de que 
doi: http://dx.doi.org/10.15359/ree.19-3.9

URL: http://www.una.ac.cr/educare

CORREO: educare@una.cr

su contribución en la escuela no sea la enseñanza de conocimientos básicos, sino la educación de los niños y de las niñas, exaltando los valores, las actitudes y los comportamientos mostrados en sus actividades cotidianas en la escuela y con sus familias. En este sentido, los saberes de la competencia de ruralidad del perfil profesional proponen, como elementos de competencia, una práctica pedagógica que sea sensible al mundo rural y la mejora del nivel educativo de las personas que habitan en el medio rural.

Como parte de las características pedagógico-comunitarias de la práctica educativa del personal docente rural, el constructivismo es parte de las fuentes filosóficas de las que se nutre la Política Educativa vigente y, aunque forma parte del acervo pedagógico de los educadores y de las educadoras de esta investigación, en las observaciones y entrevistas realizadas, no evidenciaron comprensión de los elementos caracterizadores de los supuestos teóricos y prácticos de la ideología educativa mencionada. Por lo anterior, hay desconocimiento para proyectar una apropiación de esta corriente epistemológica, sustentadora de las tareas en la práctica educativa del personal docente rural, de acuerdo con la política ministerial, lo cual invita a una reflexión crítica por parte de la academia en relación con la mediación que se realiza alrededor de las corrientes pedagógicas que son base fundamental de su formación.

La planificación didáctica se proyecta por objetivos y responde a los programas de estudio de las materias de español, matemáticas, ciencias y estudios sociales, con base en las directrices emanadas por el Ministerio de Educación Pública en materia curricular, lo que incluye algunos materiales didácticos elaborados en este Ministerio. Además, se utilizan libros de textos de editoriales privadas y ciertas propuestas que se encuentran en la "WEB", para el desarrollo de los temas. Se incorporan (cuando los contenidos lo posibilitan), elementos del contexto de la naturaleza rural. Cabe mencionar que para las personas entrevistadas, la planificación por materias no es idónea en la escuela rural, pues sugiere que el aprendizaje de las personas tiene que ser integral, ya que se asocian elementos del contexto con los contenidos de los programas. Esta tendencia de planificación didáctica es un elemento de competencia del área disciplinar y pedagógica del perfil profesional, la cual se aprende mediante los cursos de didácticas de materias específicas, la escuela multigrado y el curso de integración curricular, en consecuencia, el perfil profesional pretende que el estudiantado en formación tenga conocimientos sobre esta perspectiva curricular que no es llevada a su práctica pedagógica de forma cotidiana.

El uso de las herramientas tecnológicas, para la mediación pedagógica y la gestión administrativa, es limitado por cuanto no todas las instituciones educativas participantes poseen el equipo adecuado. Esta situación se pudo comprobar en el hecho de que todos los docentes y las docentes de esta investigación invierten sus propios recursos en computadoras portátiles o personales, que son llevadas a las escuelas, por considerarse herramientas que les permiten un quehacer más eficiente. 
doi: http://dx.doi.org/10.15359/ree.19-3.9

URL: http://www.una.ac.cr/educare

CORREO: educare@una.cr

La evaluación de los aprendizajes aplicada en la escuela rural se basa en la normativa del MEP, desde la función sumativa y formativa, mediante la construcción de instrumentos valorados en el desempeño estudiantil. Este proceso, en el caso de las escuelas unidocentes y dirección1, conlleva alta inversión de tiempo por parte de maestros y maestras. Verbigracia, para el caso de las pruebas escritas en el que se atiende de forma simultánea a los seis niveles y a las cuatro materias, se requiere de la realización de veinticuatro tablas de especificaciones para las respectivas pruebas. Esto conlleva tanto en la formación profesional como en su ejercicio, la creación de estrategias de evaluación más pertinentes y viables, lo cual visualiza una evaluación de los aprendizajes que no necesariamente se fundamente en la norma del MEP vigente, sino que se establezca una propia para para este grupo en particular.

Existe consenso, entre el grupo de participantes, sobre la importancia de propiciar un clima de organización áulico en que el proceso de enseñanza y de aprendizaje se lleve a cabo por parte de los grupos de estudiantes con orden, responsabilidad, autonomía, obediencia, independencia, silencio, confianza, esfuerzo, comunicación asertiva, colaboración y respeto entre pares. Además, se propone la realización de acciones educativas dirigidas a concienciar al estudiantado en el respeto por la naturaleza y el interés por un ambiente sostenible en la zona rural, lo cual es consecuente con los valores y actitudes que pregona el perfil profesional de la carrera en Educación Rural.

Como parte de las funciones docentes, el director o la directora de la escuela rural, como miembro de la Junta de Educación y en el Patronato Escolar, la administración de la escuela, precisa de una serie de procedimientos conjugados con la participación en reuniones del MEP, lo que significa sacrificar, de alguna u otra manera, la labor pedagógica, pues no se pueden realizar todas las acciones al mismo tiempo. Esta circunstancia es percibida por las familias del estudiantado, lo que se traduce en incomprensión por la ausencia reiterada del educador o educadora en la escuela, para impartir las lecciones. En ese sentido, no conveniente la forma en que se procede con la función administrativa en combinación con la mediación pedagógica, por lo que invoca el análisis y creación de un modelo de parte de la academia en relación con gestión escolar unidocente.

En la gestión administrativa, el personal docente participante elabora un proyecto educativo, el cual consigna las necesidades de la institución y la información recuperada, mediante el acercamiento a la familia a la escuela, y la visita, por la parte docente, a los hogares, comités y otras organizaciones de la comunidad.

Asimismo, propician un acercamiento de la familia del estudiante en la escuela, y de la comunidad en general como estrategia de apoyo para la toma de decisiones que inciden en la dinámica de la comunidad educativa. Aunque se intenta fomentar la relación escuela-familiacomunidad, los docentes y las docentes manifiestan que se da algún distanciamiento por la falta de motivación y participación de las familias, y del rol que asume el personal docente en 
doi: http://dx.doi.org/10.15359/ree.19-3.9

URL: http://www.una.ac.cr/educare

CORREO: educare@una.cr

esta relación. Se reconoce la importancia de estrechar la relación entre la escuela y la familia teniendo como eje central el beneficio hacia el estudiante.

En la gestión comunitaria, las acciones que realiza el personal docente rural van dirigidas hacia las familias y la comunidad para la búsqueda de cursos en alfabetización y en educación no formal con apoyo del MEP, del INA y de otras organizaciones no gubernamentales, así como de su participación en procesos de gestión comunitaria desde la escuela y como miembro de grupos organizados para identificar y colaborar en la solución de algunas necesidades que surgen en la comunidad. Para ello, asume una posición protagónica para convocar a la comunidad para que participe activamente en la conformación de comités como el de deportes, del agua, de protección al medio ambiente, asociación de desarrollo, entre otros, de tal manera que la comunidad se integre para la búsqueda de un mejor desarrollo de sus poblaciones.

En algunas ocasiones le corresponde liderar las reuniones de los comités u organizaciones así como elaborar solicitudes a otras instituciones con proyectos que concretan las necesidades detectadas para obtener apoyo o financiamiento que les permita ejecutarlos.

Las actitudes y valores necesarios para la docencia rural detallados por las personas entrevistadas son: la credibilidad en su propia función docente, la transparencia en su gestión administrativa y curricular, la autonomía para la toma de decisiones en beneficio de la escuela rural, la responsabilidad y el liderazgo que proyecta en la gestión comunitaria. Esta última se considera complementaria a su profesión, por cuanto conlleva acciones y oportunidades para dinamizar las comunidades en la zona rural.

En este sentido, no se consideran como ámbitos de la educación formal o de educación no formal que se asocian a potenciales laborales alternativos para el personal docente rural; sin embargo, juzgan importante que, desde la gestión comunitaria que realizan, se deben fomentar cursos en el área de educación no formal como alternativas para el desarrollo educativo en la comunidad rural.

Las posibilidades laborales en la actualidad para los docentes y las docentes rurales son limitadas en cuanto a opciones se refiere, porque aún se tiene la visión de que el único empleador es el Ministerio de Educación Pública, por lo que las personas entrevistadas ven con incertidumbre que con el mismo perfil profesional que les certifica haya apertura para otros trabajos, lo cual llama la atención para que durante la formación del profesional en educación rural se exploren otros posibles escenarios laborales.

Emergen posibles espacios laborales alternativos como el turismo rural, la organización de grupos para guía turístico, en museos, en fábricas y la pedagogía aplicada en otras áreas disciplinares. Para ello, se valoran la expresión oral, el manejo de grupos y el liderazgo como habilidades pedagógicas del perfil profesional del personal docente rural, esenciales para laborar en otros espacios. 
doi: http://dx.doi.org/10.15359/ree.19-3.9

URL: http://www.una.ac.cr/educare

CORREO: educare@una.cr

Los cursos de alfabetización para adultos continúan siendo una necesidad latente en la zona rural para aumentar el nivel educativo de la comunidad rural. Los docentes participantes la conciben como una actividad complementaria a la docencia escolar, porque cuando es posible, el MEP la incentiva con un porcentaje de salario en condición de recargo de funciones para que pueda consolidarse un grupo para desarrollar el curso que está condicionado a la matrícula inicial y permanencia del estudiantado.

\section{Referencias}

Aguilar, M. E y Angulo, L. (2007). Reseña de la División de Educación Rural. Heredia, Costa Rica. Universidad Nacional (DER-CIDE).

Antúnez, S. (2001). La actuación. En J. Gairín y P. Darder (Coords.), Organización de Centros Educativos. Aspectos básicos (pp. 329-344). Barcelona: CISSPRAXIS.

Asamblea Legislativa de la República de Costa Rica. (25 de setiembre de 1957). Ley № 2160. Ley Fundamental de Educación.

Boylan, C. R. (2003). Putting Rural Into Pre -Service Teacher Education [Poner la formación docente al servicio de la ruralidad]. Australian Association for Research in Education, Recuperado de http:// www.aare.edu.au/publications-database.php/3722/Putting-rural-into-pre--service-teachereducation

Bustos, A. (2007). Enseñar en la escuela rural aprendiendo a hacerlo. Evolución de la identidad profesional en las aulas multigrado. Profesorado. Revista de currículumy formación de profesorado, 11(3), 1-26. Recuperado de http://www.ugr.es/ recfpro/rev113COL5.pdf

De Alba, A. (1991). Evaluación curricular. Conformación conceptual del campo. México: Universidad Nacional Autónoma de México.

Dengo, M. E. (1995). Educación costarricense. San José, Costa Rica: EUNED.

Harris, M. M., Holdman, L., Clark, R. E., Harris, T. R. (2005, invierno). Rural Teachers in Project Launch. [Los docentes rurales en el lanzamiento de un proyecto]. The Rural Educador, 26(2), 23-32. Recuperado de http://www.ruraleducator.net/archive/26-2/26-2 Harris.pdf

Miller, B. A. (1991). A Review of the Qualitative Research on Multigrade Instruction [Una revisión cualitativa de la enseñanza multigrado]. Research in Rural Education, 7(2), 3-12. Recuperado de http://jrre.vmhost.psu.edu/wp-content/uploads/2014/02/7-2_4.pdf

Solano, J., Van Kampen, P., Ovares, S. (2003). El docente rural en Costa Rica: Radiografía de una profesión. Heredia, Costa Rica: EUNA. 
doi: http://dx.doi.org/10.15359/ree.19-3.9

URL: http://www.una.ac.cr/educare

CORREO: educare@una.cr

Thomas, C. y Hernández, R. (Junio, 2005). El rol del profesor en la educación rural chilena. Revista Digital eRural, Educación, cultura y desarrollo rural, 3(5). Recuperado de http://www. revistaerural.cl/thyhe.pdf

Universidad Nacional. División de Educación Rural (DER). (s. f.). Licenciatura en Educación con énfasis en Educación Rural para ly Il ciclos (Plan de estudios). Heredia, Costa Rica: Universidad Nacional. Recuperado de http://www.cide-rural.una.ac.cr/index.php?option=com_joomdoc\&task=cat_ view\&gid $=40 \& \mid$ temid $=80$

Villa, A. y Poblete, M. (Dirs.). (2007). Aprendizaje basado en competencias. Una propuesta para la evaluación de las competencias genéricas. Bilbao: Ediciones Mensajero. Recuperado de http:// delegacion233.bligoo.com.mx/media/users/20/1002571/files/240726/Aprendizaje_Basado_ en Competencias.pdf

\section{Cómo citar este artículo en APA:}

Ramírez-González, A. (Setiembre-Diciembre, 2015). Valoración del perfil docente rural desde el proceso formativo y la práctica educativa. Revista Electrónica Educare, 19(3), 1-26. doi: http://dx.doi.org/10.15359/ree.19-3.9

Nota: Para citar este artículo en otros sistemas puede consultar el hipervínculo "Como citar el artículo" en la barra derecha de nuestro sitio web: http://www.revistas.una.ac.cr/index.php/EDUCARE/index 